\title{
Formal Theory Development: From Black holes to Qubits through String Theoretic Microscopes
}

\author{
Tadashi Takayanagi* \\ Center for Gravitational Physics, Yukawa Institute for Theoretical Physics, Kyoto University \\ E-mail: takayana@yukawa.kyoto-u.ac.jp
}

\begin{abstract}
The AdS/CFT provides us a very powerful tool to study quantum gravity from the view point of quantum field theories. Even though there have been so many evidences for AdS/CFT, we still do not have a satisfactory understanding of the basic mechanism of AdS/CFT. However, owing to recent progresses, we have learned that quantum information theoretic properties such as quantum entanglement play crucial role in this basic mechanism. This leads to the idea of emergent gravity from quantum entanglement. In this talk, we would like to review these recent developments in subjects related to the AdS/CFT as one important highlight in formal theory.
\end{abstract}

The 39th International Conference on High Energy Physics (ICHEP2018)

4-11 July, 2018

Seoul, Korea

${ }^{*}$ Speaker. 


\section{Introduction}

One of the most remarkable highlights in recent formal theory developments is advanced understandings of string theory as a theory of quantum gravity. In theoretical physics, constructing theory of quantum gravity is one of the most important unsolved problems. String theory has been expected to be a complete theory of quantum gravity. However, the original approach to string theory, based on its world-sheet theory, is essentially limited to very special setups such as a flat spacetime due to partly technical and partly conceptual problems. This situation has been largely changed and improved by the discovery of the AdS/CFT correspondence [1] in 1997. This provides a concrete realization of the idea of holography $[2,3]$, namely gravitation theory in $d+2$ dimension is equivalent to non-gravitational theory (such as quantum field theories) in $d+1$ dimension. Even though we now have quite many evidences of AdS/CFT, we still do not know how it works, i.e. the basic mechanism of AdS/CFT. This has always posed us the fundamental problem of explaining the principle of AdS/CFT and this is the main reason why so many surprising progresses have been constantly produced in this subject of AdS/CFT, even after 20 years of its discovery. In particular, there have been remarkable developments on the mechanism of AdS/CFT and how the spacetime emerges from holography during these few years. Therefore we would like to talk on the development of AdS/CFT in this formal theory session.

To understand how things are going in a very tiny world, such as biology, chemistry, and condensed matter physics, we need to use various microscopes. In particle physics, the appropriate microscope is accelerators. What is a useful microscope in string theory ? If we are equipped with a correct one, we will be able to see the microscopic structure of gravity, namely quantum gravity. Actually the progresses during these twenty years tell us that a powerful microscope in string theory is the idea of holography or the AdS/CFT as its special example. Even though we cannot make actual experiments with this microscope, but it provides us with a wonderful tool when we perform thought experiments.

\section{Black Hole Entropy and String Theory}

The simplest but non-trivial laboratory for thought experiments in string theory is black holes. Indeed, black holes are a sort of condensations of the essence of gravitational theories, which is missing in any theories without gravity. One important such property of black holes is that they have entropy given by the Bekenstein-Hawking formula [4, 5]

$$
S_{B H}=\frac{A\left(\Sigma_{B H}\right)}{4 G_{N}} \text {. }
$$

This is a surprising formula because its shows that even classical theory can have non-zero entropy in gravity and also because it has a mysterious feature that the entropy is proportional not the volume but the area of a black hole. Indeed the latter property leads to the holographic principle conjecture $[2,3]$ as this formula tells us that degrees of freedom in gravity actually look like those live in lower dimension. Thus, in holography, the area law of entropy in gravity is equivalent to the familiar volume law (i.e. extenstive) property of thermodynamical entropy.

The former issue, i.e. the origin of non-zero entropy in classical gravity, has given us the strong motivation for recent studies of AdS/CFT and black hole information paradox. The explanation of 
microscopic origin of black hole entropy for supersymmetric black holes in string theory was first given in [6], where the counting microstates were performed in the holographic dual theory, namely two dimensional conformal field theories (2d CFTs). From modern understandings, this can be regarded as an application of $\mathrm{AdS}_{3} / \mathrm{CFT}_{2}$ correspondence. However, it is fair to say that the origin of black hole entropy is still not well-understood directly from the viewpoint of quantum gravity. At least this suggests us that the structure of Hilbert space for quantum gravity is so different from what we expect for local quantum field theories as is important in the arguments of firewall paradox $[7,8]$. This issue of origin of non-zero entropy in gravity gets sharper in the holographic entanglement entropy $[9,10]$, which can be regarded as a generalization of black hole entropy to generic spacetimes without black holes.

\section{AdS/CFT and Recent Developments}

The holographic principle has concrete realizations in string theory. One of the most prominent examples is the AdS/CFT correspondence [1]. The AdS/CFT correspondence argues that a gravitational theory on a $d+2$ dimensional Anti-de Sitter space $\left(\operatorname{AdS}_{d+2}\right)$ is equivalent to a conformal field theory (CFT) on the $d+1$ dimensional boundary of $\operatorname{AdS}_{d+2}$, which is simply expressed as $\mathrm{AdS}_{d+2} / \mathrm{CFT}_{d+1}$.

A typical coordinate called, the Poincare coordinate, the metric of $\mathrm{AdS}_{d+2}$ looks like by

$$
d s^{2}=R^{2} \frac{d z^{2}+d x^{\mu} d x_{\mu}}{z^{2}},
$$

where $\mu=0,1, \cdots, d$ and $R$ represents the radius of AdS. In this coordinate, the boundary of $\operatorname{AdS}_{d+2}$ is given by the spacetime spanned by $\left(x^{0}, x^{1}, \cdots, x^{d}\right)$ at $z=0$. Since the metric at $z=0$ is divergent, we introduce the cut off as $z>a$, where an infinitesimally small constant $a$. This cut off in the AdS space is equivalent to the UV cut off (or lattice spacing) $a$ in CFT up to an order one constant. The important fact is that this new coordinate $z$ describes the length scale (or inverse of energy scale) of the dual CFT in the sense of its RG-flow.

The basic principle of AdS/CFT is the bulk to boundary relation [11, 12]. This argues that the CFT partition function is equal to that of the gravity on the AdS space i.e. $Z_{C F T}=Z_{A d S}$. In the classical gravity limit, which is assumed in the most parts of this article, the gravity partition function is just given in terms of the one-shell action.

The AdS/CFT correspondence was originally discovered by considering near horizon geometries of many D-branes [13] in string theory [1]. This consideration gives the microscopic identification of CFT which is dual to string theory AdS backgrounds. The dual CFTs are typically given by $S U(N)$ Yang-Mills gauge theories with a ('t Hooft) coupling constant $\lambda$, corresponding to $N$ D-branes. In the most famous setup: $\operatorname{AdS}_{5} \times S^{5}$, we find that the raito $R / l_{s t}$ and $R / l_{p l}$ is proportional to $\lambda^{1 / 4}$ and $N^{1 / 4}$, where $l_{s t}$ and $l_{p l}$ are the string length and Planck length. Therefore classical gravity limit (or called supergravity limit) is given by the limit where both $N$ and $\lambda$ are infinitely large. This is because the large $N$ limit suppresses quantum gravity effects and the strong coupling limit suppresses the string theoretic corrections.

The AdS/CFT can be applied to more general backgrounds. We can modify the infrared (IR) geometry i.e. the large $z$ region. We always require that in the boundary limit $z \rightarrow 0$, the metric approaches to that of the pure AdS (3.1). Such spacetimes are called asymptotically AdS. 
The AdS/CFT have been applied to various strong couples quantum systems such as QCD and condensed matter physics. Such applied AdS/CFT direct was pioneered by the calculation of shear viscosity $\eta$, which showed that the ratio $\eta / s$ of shear viscosity versus entropy density takes a universal value

$$
\frac{\eta}{s}=\frac{1}{4 \pi}
$$

in holographic calculations [14]. This estimation explained the smallness of shear viscosity in quark-gluon plasma in a semi-quantitative way. This implies that CFTs with classical gravity duals, so called holographic CFTs, capture a universal feature of extremely strongly coupled condensed matter systems. More recently, another universal aspect of holographic CFTs has been uncovered: holographic CFTs are maximally chaotic in the sense of quantum chaos. A useful quantitative measure of chaos is the Lyapunov exponent $\lambda_{L}$ and it was proved that in holographic CFTs, Lyapunov exponent takes the maximal value:

$$
\lambda_{L}=2 \pi T
$$

where $T$ is the temperature of the quantum system.

\section{Emergent Spacetime from Quantum Entanglement}

Even though we have plenty of examples where the AdS/CFT have been successfully applied, we still do not fully understand the fundamental mechanism of AdS/CFT. To get into this deep problem, it looks very important to study how the information in the CFT is encoded in that in the gravity theory. Especially, we can consider the amount of information included in a certain region $A$ in the CFT and ask what is dual to it in the AdS gravity. Since the amount of information in the region $A$ can be measured by the quantity called entanglement entropy $A$, it will be interesting to consider what is the AdS dual of the entanglement entropy in a CFT.

\subsection{Definition and Properties of Entanglement Entropy}

A state in quantum mechanics is described by a vector $|\Psi\rangle$ in a given Hilbert space $\mathscr{H}$, which evolves in time by the Hamiltonian $H$. We assume the quantum system we consider has multiple degrees of freedom so that we can decompose the total system into two subsystems $A$ and $B$. Then the total Hilbert space $\mathscr{H}$ becomes a direct products

$$
\mathscr{H}=\mathscr{H}_{A} \otimes \mathscr{H}_{B}
$$

In quantum mechanics, physical quantities are computed as expectation values of operators: $O\rangle=\langle\Psi|O| \Psi\rangle=\operatorname{Tr}[\rho \cdot O]$, where we defined the density matrix $\rho=|\Psi\rangle\langle\Psi|$. Such a system is called a pure state as it is described by a unique wave function $|\Psi\rangle$. In more general cases, called mixed states, the system is described by a density matrix $\rho$ itself, normalized such that $\operatorname{Tr} \rho=1$. A typical example of a mixed state is the canonical distribution $\rho=e^{-\beta H} / \operatorname{Tr}\left[e^{-H / T}\right]$.

Next we introduce the reduced density matrix $\rho_{A}$ for the subsystem $A$ by tracing out with respect to $\mathscr{H}_{B}$ as $\rho_{A}=\operatorname{Tr}_{B}[\rho]$. Then the entanglement entropy is defined as the von-Neumann entropy for $\rho_{A}$

$$
S_{A}=-\operatorname{Tr}\left[\rho_{A} \log \rho_{A}\right]
$$


In this talk, we consider the entanglement entropy in quantum field theories (QFTs). Refer to $[16,17]$ for excellent reviews on this topic. We can view a QFT as an infinite copies of quantum mechanics. Thus, its Hilbert space $\mathscr{H}$ is given by all possible field configurations of QFT at a fixed time. Since the choice of $A$ is uniquely defined in terms of the boundary $\partial A$, there are obviously infinitely different definitions of the entanglement entropy $S_{A}$ depending on the choices of $A$.

We summarize the basic properties of the entanglement entropy. If the total system is pure, the equality $S_{A}=S_{B}$ is always satisfied. Also, for any systems, when we decompose the system into four subsystems $A, B, C$ and $D$ so that there are no overlap between each of them i.e. $\mathscr{H}=$ $\mathscr{H}_{\mathscr{A}} \otimes \mathscr{H}_{\mathscr{B}} \otimes \mathscr{H}_{\mathscr{C}} \otimes \mathscr{H}_{\mathscr{D}}$, the following inequality is always satisfied:

$$
S_{A \cup B}+S_{B \cup C} \geq S_{A \cup B \cup C}+S_{B}
$$

This inequality is called the strong subadditivity.

One more useful property is the area law for QFTs. Since QFTs have infinitely many degrees of freedom, the entanglement entropy $S_{A}$ gets divergent. We can show that the leading divergent term is proportional to the area of the boundary $\partial A$ :

$$
S_{A}=\gamma \cdot \frac{\operatorname{Area}(\partial A)}{a^{d-1}}+O\left(a^{-(d-2)}\right),
$$

where $\gamma$ is a numerical constant; $a$ is the ultra-violet(UV) cut off or equally the lattice constant. This property is called the area law. We should note that there is an important exception of area law (4.4). In $2 \mathrm{~d}$ CFTs, the area law is violated in a logarithmic way. When $A$ is an length $L$ interval, $S_{A}$ is universally given by

$$
S_{A}=\frac{c}{3} \log \frac{l}{a},
$$

where $c$ is the central charge of the CFT.

\subsection{Holographic Entanglement Entropy}

Now we would like to turn to holographic calculations of entanglement entropy. As found in [9], the entanglement entropy $S_{A}$ for a holographic CFT is computed from the area of minimal area surface

$$
S_{A}=\operatorname{Min}_{\Sigma_{A}}\left[\frac{\operatorname{Area}\left(\Sigma_{A}\right)}{4 G_{N}}\right]
$$

where $\Sigma_{A}$ is a codimension two surface (i.e. $d$ dimensional in $\mathrm{AdS}_{d+2}$ ) which satisfies $\partial \Sigma_{A}=\partial A$; in addition we require that $\Sigma_{A}$ is homologous to $A$. The minimum in (4.6) should be taken for all surfaces $\Sigma_{A}$ which satisfy this condition. This formula (4.6) can be applied to any static setups. The minimal area surface is well-defined in the static case because we can equivalently consider a Euclidean AdS space.

When a given background is time-dependent, we need to use instead the covariant holographic entanglement entropy [10], which is given by replacing $\Sigma_{A}$ with the extremal surface in the Lorentzian asymptotic AdS space which satisfies the previous condition.

The derivation of holographic entanglement entropy has been given in [18, 19, 20], based on the bulk-boundary relation of AdS/CFT [11, 12]. It is intriguing to note that we can derive quantum 
information theoretical inequalities from geometric arguments using the holographic entanglement entropy. such as the proof of strong subadditivity (4.3) [21].

The entanglement entropy have a useful dynamical property called the first law, which is analogous to the first law of thermodynamics. It is the property that when we slightly excite a quantum system, the entanglement entropy is increased such that it is proportional to a certain energy. This was first found in [23] for holographic systems. The energy is called modular Hamiltonian, given by $H_{A}=-\log \rho_{A}$ and the first law is written as $\Delta S_{A} \simeq \Delta H_{A}$ [23]. Interestingly it have turned our that this first law is equivalent to the linearized part of the Einstein equation [24, 25]. This suggests that the dynamics of quantum entanglement is equivalent to that of gravity. Generalizations of this argument to higher non-linear orders have recently been performed [26, 27].

Let us come back to the original question in AdS/CFT: which part in AdS is dual to which part in CFT ? The holographic entanglement entropy formula strongly suggests that the region surrounded by $\Sigma_{A}$ and $A$, which is called entanglement wedge, is dual to the reduced density matrix $\rho_{A}$. This speculation has been justified with more rigorous arguments in $[28,29]$ using a quantity called relative entropy.

This entanglement wedge provided us a new interesting geometric object in AdS/CFT. We expect that its cross section area is an important quantity. Indeed, it was conjectured that this entanglement wedge cross section, divided by $4 G_{N}$, is dual to a quantity called entanglement of purification, which is a useful measure of correlations between two systems [30, 31].

\subsection{Emergent Spacetime from Quantum Entanglement and Tensor Networks}

The holographic entanglement entropy tells us that the amount of quantum entanglement corresponds to the area in the gravity dual. This suggests the spacetime in gravity may emerge from quantum entanglement. One way to realize this idea is to employ so called tensor networks, which is a network description of a quantum state in a many-body system, first proposed in [32] (see also other approaches $[33,341)$. One well-known class of tensor network which are dual to CFTs is called MERA (Multi-scale Entanglement Renormalization Ansatz) [35]. Recently, it was pointed out the quantum error correcting codes, which is a strong method to protect the data against noise in quantum computing, is closed related to the mechanism realization of the bulk AdS from a CFT [36]. By using this idea, a new tensor network called perfect tensor networks, have been constructed [37]. This provides us with an ideal toy model of AdS/CFT.

In these connection between the tensor networks and AdS/CFT, the quantum state is described by a network of tensors. This network describes how the system is entangled and adds an extra dimension, eventually leads to a time slice of AdS spacetime. This nicely reproduces the holographic entanglement entropy formula (4.6). However, the presence of time direction is not manifest in such a tensor network description. Nevertheless, we can describe how the network geometry changes under the time evolution from the information of CFT Hamiltonian, which is indeed similar to the propagation of gravitational waves [38].

A systematical formulation of this AdS/tensor network duality is summarized into two principles: (i) a codimension two space-like surface is dual to a quantum state in a holographic CFT (called surface/state correspondence) [39], and (ii) a codimension one (both time-like and spacelike slice ) is dual to a quantum circuit defined by a path-integration on the slice with the Lagrangian given by an appropriate coarse-graining of the CFT [40]. The latter property is explained by the 
fact that the wave functional in CFTs is invariant under the Weyl transformation of the background metric, called path-integral optimization [41].

\section{Conclusions}

In this talk, we have reviewed recent developments in formal theory by focusing on the subjects related to the AdS/CFT. Even though we still do not have clear understandings of the basic mechanism of AdS/CFT, we have made remarkable progresses in particular, using the quantum information theoretic viewpoints. To proceed more we need to understand the connection between the AdS/CFT and tensor networks in a more concrete way. For example, we need to efficiently take into account the strongly coupled and large $N$ nature of holographic CFTs in this framework. This connection to tensor networks is also closed related to the calculation of computational complexity. This quantity is defined by the minimal number of quantum gates which transform a simple state (e.g. the direct product state) into a state which we want (e.g. the ground state of CFT). Recently an interesting holographic formula which estimates the complexity for CFT states was proposed [42]. In the tensor network description, we can estimate the complexity by counting the number of tensors. Therefore, it is natural to expect that we may have a direct connection between gravity and tensor networks through studies of complexity. The first step for this approach was taken in the recent work [41] and it will be important to extract special features for holographic CFTs which explain the mechanism of AdS/CFT.

\section{References}

[1] J. M. Maldacena, "The Large N limit of superconformal field theories and supergravity," Int. J. Theor. Phys. 38 (1999) 1113 [Adv. Theor. Math. Phys. 2 (1998) 231] doi:10.1023/A:1026654312961, 10.4310/ATMP.1998.v2.n2.a1 [hep-th/9711200].

[2] G. 't Hooft, "Dimensional reduction in quantum gravity," Conf. Proc. C 930308 (1993) 284 [gr-qc/9310026].

[3] L. Susskind, “The World as a hologram," J. Math. Phys. 36 (1995) 6377 doi:10.1063/1.531249 [hep-th/9409089].

[4] J. D. Bekenstein, "Black holes and entropy," Phys. Rev. D 7 (1973) 2333. doi:10.1103/PhysRevD.7.2333

[5] S. W. Hawking, "Particle Creation by Black Holes," Commun. Math. Phys. 43 (1975) 199 Erratum: [Commun. Math. Phys. 46 (1976) 206]. doi:10.1007/BF02345020, 10.1007/BF01608497

[6] A. Strominger and C. Vafa, "Microscopic origin of the Bekenstein-Hawking entropy," Phys. Lett. B 379 (1996) 99 doi:10.1016/0370-2693(96)00345-0 [hep-th/9601029].

[7] A. Almheiri, D. Marolf, J. Polchinski and J. Sully, "Black Holes: Complementarity or Firewalls?," JHEP 1302 (2013) 062 doi:10.1007/JHEP02(2013)062 [arXiv:1207.3123 [hep-th]].

[8] K. Papadodimas and S. Raju, "State-Dependent Bulk-Boundary Maps and Black Hole Complementarity," Phys. Rev. D 89 (2014) no.8, 086010 doi:10.1103/PhysRevD.89.086010 [arXiv:1310.6335 [hep-th]].

[9] S. Ryu and T. Takayanagi, "Holographic derivation of entanglement entropy from AdS/CFT," Phys. Rev. Lett. 96 (2006) 181602 doi:10.1103/PhysRevLett.96.181602 [hep-th/0603001]. 
[10] V. E. Hubeny, M. Rangamani and T. Takayanagi, "A Covariant holographic entanglement entropy proposal," JHEP 0707 (2007) 062 [arXiv:0705.0016 [hep-th]].

[11] S. S. Gubser, I. R. Klebanov and A. M. Polyakov, "Gauge theory correlators from noncritical string theory," Phys. Lett. B 428 (1998) 105 doi:10.1016/S0370-2693(98)00377-3 [hep-th/9802109].

[12] E. Witten, “Anti-de Sitter space and holography," Adv. Theor. Math. Phys. 2 (1998) 253 doi:10.4310/ATMP.1998.v2.n2.a2 [hep-th/9802150].

[13] J. Polchinski, “Dirichlet Branes and Ramond-Ramond charges," Phys. Rev. Lett. 75 (1995) 4724 doi: 10.1103/PhysRevLett.75.4724 [hep-th/9510017].

[14] P. Kovtun, D. T. Son and A. O. Starinets, "Viscosity in strongly interacting quantum field theories from black hole physics," Phys. Rev. Lett. 94 (2005) 111601 doi:10.1103/PhysRevLett.94.111601 [hep-th/0405231].

[15] J. Maldacena, S. H. Shenker and D. Stanford, "A bound on chaos," JHEP 1608 (2016) 106 doi:10.1007/JHEP08(2016)106 [arXiv:1503.01409 [hep-th]].

[16] P. Calabrese and J. Cardy, "Entanglement entropy and conformal field theory," J. Phys. A 42 (2009) 504005 doi:10.1088/1751-8113/42/50/504005 [arXiv:0905.4013 [cond-mat.stat-mech]].

[17] H. Casini and M. Huerta, "Entanglement entropy in free quantum field theory," J. Phys. A 42 (2009) 504007 doi:10.1088/1751-8113/42/50/504007 [arXiv:0905.2562 [hep-th]].

[18] A. Lewkowycz and J. Maldacena, “Generalized gravitational entropy,” JHEP 1308 (2013) 090 doi: 10.1007/JHEP08(2013)090 [arXiv:1304.4926 [hep-th]].

[19] T. Faulkner, A. Lewkowycz and J. Maldacena, "Quantum corrections to holographic entanglement entropy," JHEP 1311 (2013) 074 doi:10.1007/JHEP11(2013)074 [arXiv:1307.2892 [hep-th]].

[20] X. Dong, A. Lewkowycz and M. Rangamani, "Deriving covariant holographic entanglement," JHEP 1611 (2016) 028 doi:10.1007/JHEP11(2016)028 [arXiv:1607.07506 [hep-th]].

[21] M. Headrick and T. Takayanagi, "A Holographic proof of the strong subadditivity of entanglement entropy,” Phys. Rev. D 76 (2007) 106013 doi:10.1103/PhysRevD.76.106013 [arXiv:0704.3719 [hep-th]].

[22] J. Bhattacharya, M. Nozaki, T. Takayanagi and T. Ugajin, "Thermodynamical Property of Entanglement Entropy for Excited States," Phys. Rev. Lett. 110 (2013) no.9, 091602 doi:10.1103/PhysRevLett.110.091602 [arXiv:1212.1164 [hep-th]].

[23] D. D. Blanco, H. Casini, L. Y. Hung and R. C. Myers, "Relative Entropy and Holography," JHEP 1308 (2013) 060 doi:10.1007/JHEP08(2013)060 [arXiv:1305.3182 [hep-th]].

[24] N. Lashkari, M. B. McDermott and M. Van Raamsdonk, "Gravitational dynamics from entanglement 'thermodynamics'," JHEP 1404 (2014) 195 doi:10.1007/JHEP04(2014)195 [arXiv:1308.3716 [hep-th]].

[25] T. Faulkner, M. Guica, T. Hartman, R. C. Myers and M. Van Raamsdonk, "Gravitation from Entanglement in Holographic CFTs,” JHEP 1403 (2014) 051 doi:10.1007/JHEP03(2014)051 [arXiv:1312.7856 [hep-th]].

[26] T. Faulkner, F. M. Haehl, E. Hijano, O. Parrikar, C. Rabideau and M. Van Raamsdonk, "Nonlinear Gravity from Entanglement in Conformal Field Theories," JHEP 1708 (2017) 057 doi:10.1007/JHEP08(2017)057 [arXiv:1705.03026 [hep-th]]. 
[27] G. Sarosi and T. Ugajin, "Modular Hamiltonians of excited states, OPE blocks and emergent bulk fields," JHEP 1801 (2018) 012 doi:10.1007/JHEP01(2018)012 [arXiv:1705.01486 [hep-th]].

[28] D. L. Jafferis, A. Lewkowycz, J. Maldacena and S. J. Suh, "Relative entropy equals bulk relative entropy," JHEP 1606 (2016) 004 doi:10.1007/JHEP06(2016)004 [arXiv:1512.06431 [hep-th]].

[29] X. Dong, D. Harlow and A. C. Wall, "Reconstruction of Bulk Operators within the Entanglement Wedge in Gauge-Gravity Duality," Phys. Rev. Lett. 117 (2016) no.2, 021601 doi:10.1103/PhysRevLett.117.021601 [arXiv:1601.05416 [hep-th]].

[30] K. Umemoto and T. Takayanagi, "Entanglement of purification through holographic duality," Nature Phys. 14 (2018) no.6, 573 doi:10.1038/s41567-018-0075-2 [arXiv:1708.09393 [hep-th]].

[31] P. Nguyen, T. Devakul, M. G. Halbasch, M. P. Zaletel and B. Swingle, "Entanglement of purification: from spin chains to holography," JHEP 1801 (2018) 098 doi:10.1007/JHEP01(2018)098 [arXiv:1709.07424 [hep-th]].

[32] B. Swingle, "Entanglement Renormalization and Holography," Phys. Rev. D 86, 065007 (2012), arXiv:0905.1317 [cond-mat.str-el].

[33] M. Van Raamsdonk, "Building up spacetime with quantum entanglement," Gen. Rel. Grav. 42 (2010) 2323 [Int. J. Mod. Phys. D 19 (2010) 2429] doi:10.1007/s10714-010-1034-0, $10.1142 / \mathrm{S} 0218271810018529$ [arXiv: 1005.3035 [hep-th]].

[34] J. Maldacena and L. Susskind, "Cool horizons for entangled black holes," Fortsch. Phys. 61 (2013) 781 doi:10.1002/prop.201300020 [arXiv:1306.0533 [hep-th]].

[35] G. Vidal,"A class of quantum many-body states that can be efficiently simulated," Phys. Rev. Lett. 101, 110501 (2008) , arXiv:quant-ph/0610099; “Entanglement renormalization,” Phys. Rev. Lett. 99, 220405 (2007) , arXiv:cond-mat/0512165.

[36] A. Almheiri, X. Dong and D. Harlow, "Bulk Locality and Quantum Error Correction in AdS/CFT," JHEP 1504 (2015) 163 doi:10.1007/JHEP04(2015)163 [arXiv:1411.7041 [hep-th]];

[37] F. Pastawski, B. Yoshida, D. Harlow and J. Preskill, "Holographic quantum error-correcting codes: Toy models for the bulk/boundary correspondence," JHEP 1506 (2015) 149 doi:10.1007/JHEP06(2015)149 [arXiv:1503.06237 [hep-th]].

[38] M. Nozaki, S. Ryu and T. Takayanagi, "Holographic Geometry of Entanglement Renormalization in Quantum Field Theories," JHEP 1210 (2012) 193 doi:10.1007/JHEP10(2012)193 [arXiv:1208.3469 [hep-th]]; A. Mollabashi, M. Nozaki, S. Ryu and T. Takayanagi, "Holographic Geometry of cMERA for Quantum Quenches and Finite Temperature," JHEP 1403 (2014) 098 doi:10.1007/JHEP03(2014)098 [arXiv:1311.6095 [hep-th]].

[39] M. Miyaji and T. Takayanagi, "Surface/State Correspondence as a Generalized Holography," PTEP 2015 (2015) 7, 073B03 doi:10.1093/ptep/ptv089 [arXiv:1503.03542 [hep-th]].

[40] T. Takayanagi, "Holographic Spacetimes as Quantum Circuits of Path-Integrations," arXiv: 1808.09072 [hep-th].

[41] P. Caputa, N. Kundu, M. Miyaji, T. Takayanagi and K. Watanabe, "Anti-de Sitter Space from Optimization of Path Integrals in Conformal Field Theories," Phys. Rev. Lett. 119 (2017) no.7, 071602 doi:10.1103/PhysRevLett.119.071602 [arXiv:1703.00456 [hep-th]].

[42] A. R. Brown, D. A. Roberts, L. Susskind, B. Swingle and Y. Zhao, "Holographic Complexity Equals Bulk Action?," Phys. Rev. Lett. 116 (2016) no.19, 191301 doi:10.1103/PhysRevLett.116.191301 [arXiv:1509.07876 [hep-th]]; 